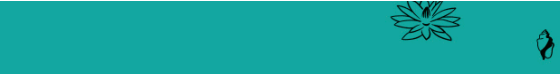

DESARROLLO Y

$y^{3}$

* DERECHO AL की

DESARROLLO

Desde el biocentrismo y
elpensamiento complejo

粠

Bernardo Alfredo Hernández Umaña

龊

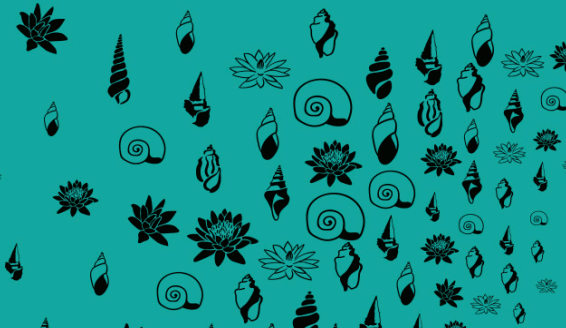

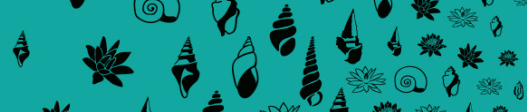

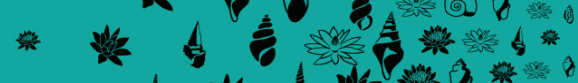

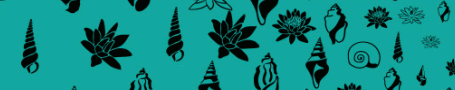

8 \& 6 (1)

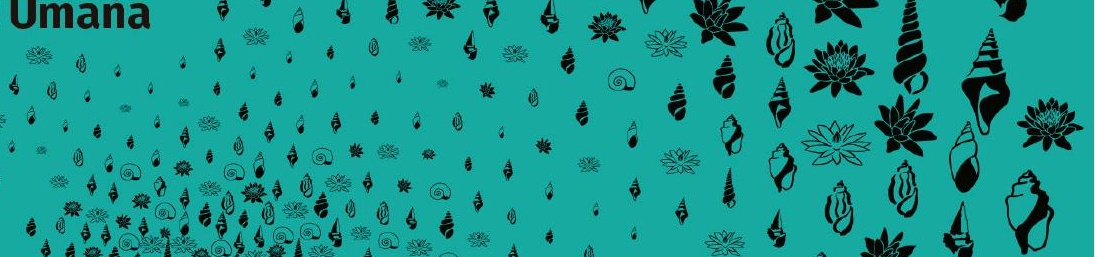

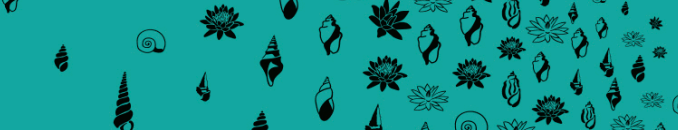

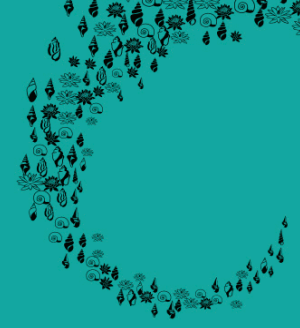

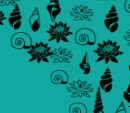

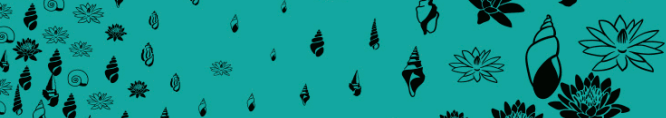

Q.

of

จ

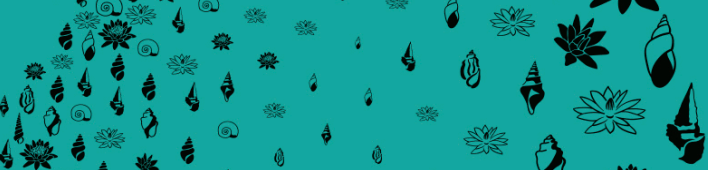

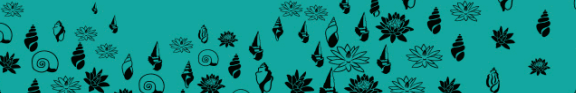

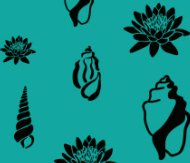

i

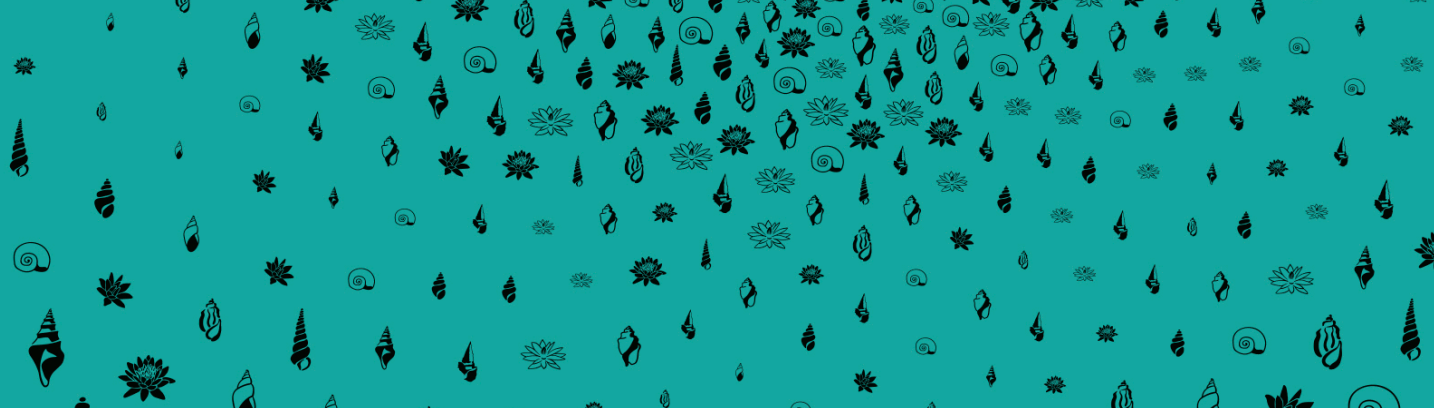

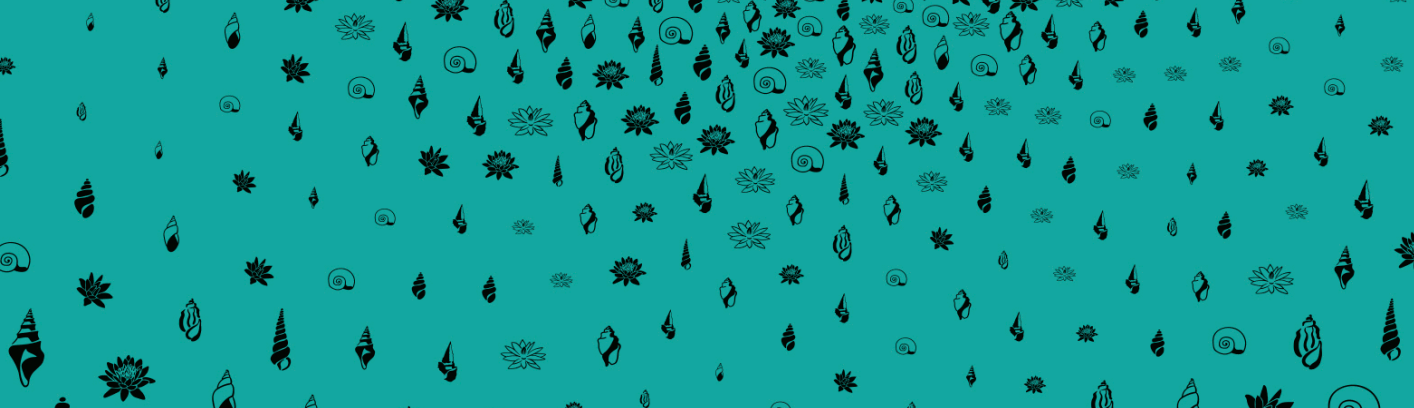

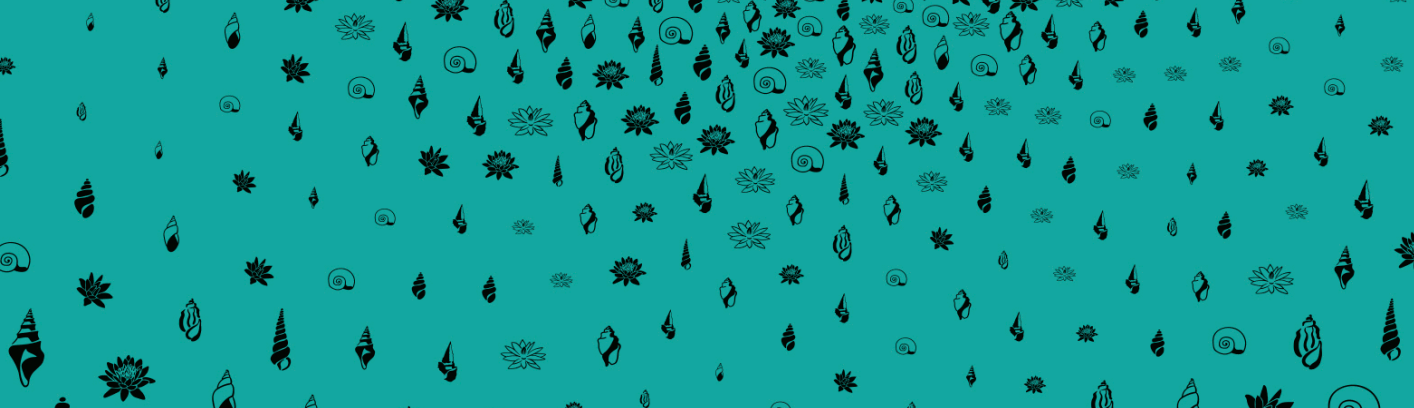

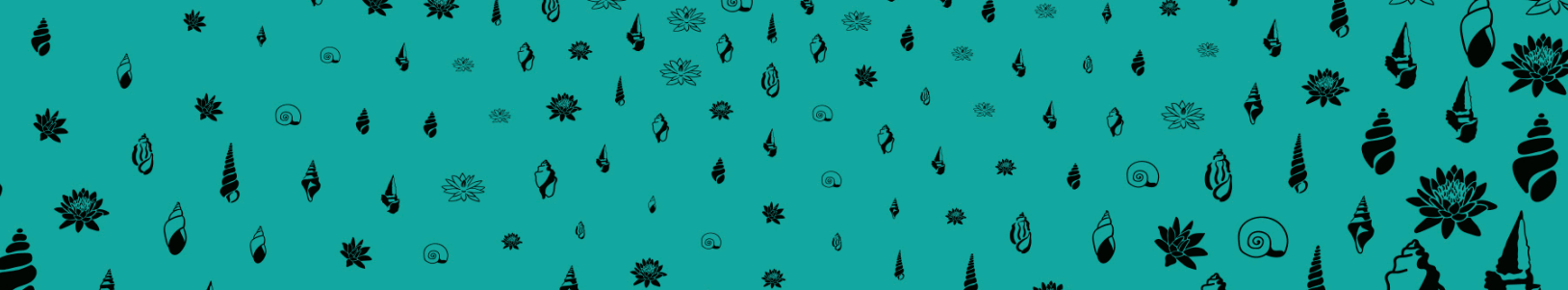

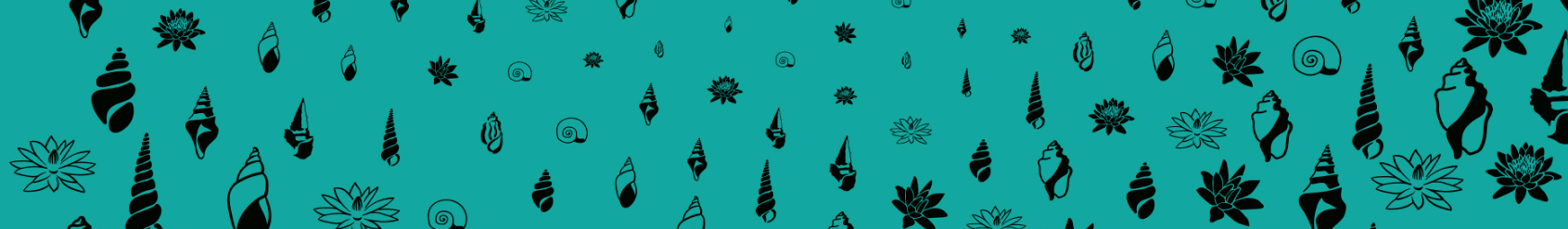

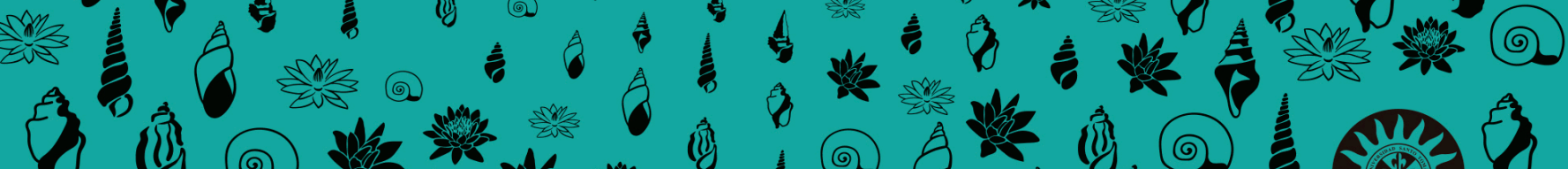

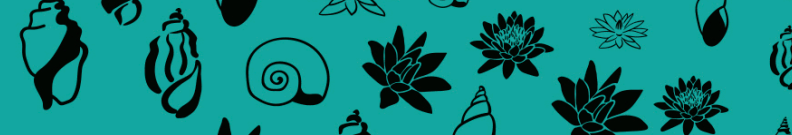
(a) (ब)

(ii) (0) (0)

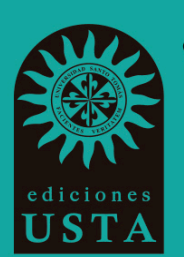

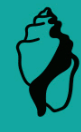




\section{Desarrollo y derecho al desarrollo}

\section{Desde el biocentrismo}

y el pensamiento complejo

Bernardo Alfredo Hernández Umaña 


\section{Desarrollo y derecho al desarrollo}

\section{Desde el biocentrismo}

y el pensamiento complejo

Bernardo Alfredo Hernández Umaña

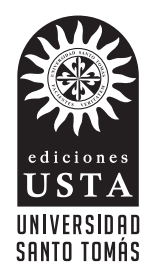


Hernández Umaña, Bernardo Alfredo

Desarrollo y derecho al desarrollo: Desde el biocentrismo y el pensamiento complejo/ Bernardo Alfredo Hernández Umaña, Bogotá: Universidad Santo Tomás, 2017.

xxv, 423 páginas; ilustraciones

Incluye referencias bibliográficas (páginas 399-423)

ISBN: 978-958-782-057-7

1. Derechos humanos 2. Desarrollo humano 3. Filosofía -- Ética I. Universidad Santo Tomás (Colombia).

CDD 340.11

CO-BoUST

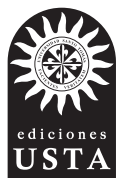

(C) Bernardo Alfredo Hernández-Umaña

(C) Universidad Santo Tomás

Ediciones USTA Carrera 9 N. ${ }^{\circ}$ 51-11

Bogotá, D. C., Colombia

Teléfonos: (+571) 5878797 ext. 2991

editorial@usantotomas.edu.co

http://ediciones.usta.edu.co

Coordinación de libros: Karen Grisales Velosa

Asistencia editorial: Andrés Felipe Andrade Cañón

Corrección de estilo: Irina Florián

Diagramación: Javier Barbosa

Diseño de cubierta: Kilka Diseño Gráfico

Hecho el depósito que establece la ley

ISBN: 978-958-782-057-7

e-ISBN: 978-958-782-058-4

Impreso en Colombia • Printed in Colombia

Impreso por: Grupo Dao Digital, S.A.S.

Primera edición, 2017

Todos los derechos reservados.

Se probíbe la reproducción total o parcial de esta obra, por cualquier medio, sin la autorización expresa del titular de los derechos.

Impreso en Colombia • Printed in Colombia 
A la Conciencia Suprema, por haber permitido que este libro viera la luz 


\section{AGRADECIMIENTOS}

Doy infinitas gracias a la Conciencia Suprema que en todo momento me ha acompañado y ha permitido que este libro viera la luz.

A mi familia por su apoyo y a todas aquellas personas que extendieron su mano y me brindaron el tiempo necesario, consejos y sugerencias que me llevaron a la reflexión, y que hoy en gran medida se reflejan en la culminación de este libro. 


\section{Índice}

Prólogo XV

INTRODUCCIÓN

Primera parte. Del desarrollo y Derecho

AL DESARROLLO COMO DERECHO HUMANO 27

Capítulo I. El concepto de desarrollo.

Hacia una realidad compleja $\quad 29$

$\begin{array}{ll}\text { Contexto y visión tradicional } & 30\end{array}$

La moda verde del desarrollo sostenible $\quad 34$

El desarrollo a escala humana y el desarrollo humano 43

Acerca de los objetivos de desarrollo del milenio y la agenda de desarrollo pos-2015 50

¿Y qué se dice desde la ética del desarrollo? $\quad 52$

El posdesarrollo $\quad 63$

Decrecimiento $\quad 67$

$\begin{array}{ll}\text { Etno-desarrollo } & 72\end{array}$

$\begin{array}{ll}\text { Co-desarrollo } & 73\end{array}$

Enfoque de género en el desarrollo $\quad 75$

$\begin{array}{ll}\text { Trans-desarrollo } & 76\end{array}$

Aportaciones del profesor Koldo Unceta para definir el desarrollo $\quad 77$

¿El Sumak Kawsay y Suma Qamaña

nos orientan hacia el Buen Vivir? $\quad 79$

Principio femenino y el mal desarrollo $\quad 85$

$\begin{array}{ll}\text { Nuestra posición } & 88\end{array}$

Capítulo II. El derecho al desarrollo en

el catálogo de los derechos humanos $\quad 91$

La generación de los nuevos derechos o derechos emergentes 92

Críticas a la tercera generación de derechos 96 
Reconocimiento del derecho al desarrollo

como derecho humano por la comunidad internacional

Críticas y observaciones respecto al reconocimiento

del derecho al desarrollo como derecho humano

Titularidad, deberes y configuración conceptual

del derecho al desarrollo

Segunda PARTe. De la fundamentación

DEL DERECHO AL DESARROLLO

I 4 I

Capítulo III. ¿Existe algún planteamiento que dé cuenta de la fundamentación del derecho al desarrollo?

La idea de la dignidad humana y libre desarrollo

de la personalidad que se deriva del pensamiento kantiano

La dignidad como bien fundamental en

la teoría de la justicia de John Rawls

El desarrollo como libertad según Amartya Sen

166

El enfoque de capacidades según Martha Nussbaum

Capítulo IV. La espiral de Arquímedes. Antropocentrismo, biocentrismo, responsabilidad y solidaridad

Sección primera Entre antropocentrismos y biocentrismos

Introducción en la fundamentación

ética del derecho al desarrollo

El antropocentrismo fuerte

El antropocentrismo débil o moderado

El biocentrismo débil

El biocentrismo fuerte o extremo

Sección segunda. Entre responsabilidad y solidaridad 256

$\begin{array}{ll}\text { Acerca de la responsabilidad } & 256\end{array}$

Acerca de la solidaridad

Tercera PARTE. ComplejIDAd, PENSAMIENTO Y ÉTICA COMPLEJA: FUNDAMENTO ÉTICO DEL DERECHO AL DESARROLLO

Capítulo V. Complejidad, pensamiento y ética compleja: 
La complejidad

El pensamiento complejo de Edgar Morin

La ética social de la vida de François Houtart 304

Crítica al pensamiento complejo de Morin 307

La dialéctica del medio de Françoise Ost 312

La unidad dialéctica de Vandana Shiva $\quad 314$

Ética de la complejidad de Edgar Morin 316

Una aproximación al fundamento ético del derecho

al desarrollo de la Comunidad de la Vida desde la complejidad

Capítulo VI. Biociudadanía y núcleo esencial del derecho al desarrollo de la Comunidad de la Vida

Sección primera. Biociudadanía

Biociudadanía

Sección segunda. El núcleo esencial

del derecho al desarrollo de la Comunidad de la Vida

¿Por qué hablar de un núcleo esencial?

Propuesta de núcleo esencial del derecho al desarrollo

Conclusiones

Epílogo

La Biociudadanía

REFERENCIAS BIBLIOGRÁFICAS 


\section{Prólogo}

Este libro recoge en lo fundamental la tesis doctoral titulada El derecho Cal desarrollo: fundamentación ética, biociudadanía y núcleo esencial, presentada a la Facultad de Derecho de la Universidad Complutense de Madrid, en el marco del Doctorado en Derecho, el 12 de junio de 2015 bajo la dirección del Doctor José Iturmendi Morales, Director del Departamento de Filosofía del Derecho, Moral y Política I, de la Universidad Complutense de Madrid y defendida ante el Tribunal conformado por la Doctora María José Falcón Tella de la Universidad Complutense de Madrid, presidenta; la Doctora Isabel Hoyo Sierra de la Universidad Rey Juan Carlos, primer vocal; el Doctor Santiago Carretero Sánchez de la Universidad Rey Juan Carlos, segundo vocal; el Doctor Enrico Pascucci de Ponte de la Universidad Alfonso X el Sabio, tercer vocal y el Doctor Juan Antonio Martínez Muñoz de la Universidad Complutense de Madrid, secretario, quienes otorgaron la calificación "sobresaliente cum laude por unanimidad".

Además, es el resultado de la dedicación, el esfuerzo permanente y la reflexión que, a lo largo de varios años he realizado en torno al desarrollo y el derecho al desarrollo en la Comunidad de la Vida, que finalmente ha visto la luz en esta obra, y pretende ser un faro que ilumina el camino de la construcción del conocimiento complejo, y aporta a la discusión y al encuentro de saberes desde todas las orillas posibles.

I 2 DE MAYO DE 20I7. Bogotá, D.C, Colombia.

EL AUTOR. 


\section{Introducción}

T a comprensión del desarrollo sólo como crecimiento económico, Lle imprime un cariz que lo limita al plano meramente material y lo hace incompleto, teniendo en cuenta que éste mide, entre otros, el Producto Interno Bruto ${ }^{1}$ (PIB); que en el presente, no es representativo en el desarrollo del ser humano, ni del medio ambiente, sino, del aumento de la riqueza de una nación, solamente crecen los medios más no el fin. No obstante, hay algunos Estados que han utilizado el medio en el fin y ello se ha visto reflejado en el acceso de oportunidades, y una mejor calidad de vida en sus ciudadanos. Especialmente en los denominados países desarrollados.

Destacamos que en el Informe Brundtland de 1987, se empezaba a vislumbrar la necesidad de replantear el modelo de desarrollo, que ha estado distanciado de la relación entre la naturaleza y el ser humano, este documento llevó como título Nuestro futuro común y fue uno de los puntos de partida para hablar del desarrollo sostenible.

1 Es el valor total de la producción corriente de bienes y servicios finales dentro del territorio nacional durante un período de tiempo que generalmente es un trimestre o un año. 
En el escenario del desarrollo, la libertad y la creación de capacidades ha sido un camino que ha contado con el beneplácito de la comunidad internacional. Así se ha expresado en declaraciones y resoluciones en las cuales se menciona el interés y la apuesta por crear capacidades a partir de la libertad para lograr el desarrollo, pero aún sigue sin completarse el engranaje que esta sinergia requiere. Pues la idea es que realmente los medios sean los medios y nos centremos en lo fundamental, el ser humano y a la vez en la Comunidad de la Vida, y no en el crecimiento de las economías de los Estados, y de los intereses de algunos privilegiados.

En la evolución conceptual del desarrollo no ha faltado el deseo de adicionarle adjetivos con la intención de centrar la atención en lo fundamental, como la naturaleza y el ser humano, sin embargo, en aras de definirlo ha sido un desgaste innecesario. Para los tiempos que marchan es necesario comprender que, sea cualquier adjetivo que se le agregue al desarrollo, éste no dejará de ser desarrollo y debemos concebirlo con una visión compleja que integre a la Comunidad de la Vida.

De otra parte, cuando se habla del derecho al desarrollo, nos enfrentamos ante un derecho catalogado como humano que, al ser de reciente aparición —en la década de los años setenta del siglo pasado-, se ha estudiado especialmente desde una óptica, el derecho internacional (Gómez, 1999). Con lo cual se ha dejado de lado la necesaria fundamentación ética que necesita este derecho para aportarle otra visión, como al tener en cuenta a la Madre Tierra y al ser humano, y poder hacer una comprensión diferente del desarrollo, del derecho al desarrollo y su realización.

Parecería razonable que hubiera cierta armonía entre lo que es aceptado en la comunidad internacional por desarrollo y derecho al desarrollo, pero no la hay. Se ha suscitado un sin número de propuestas novedosas en torno al desarrollo, pero estas desbordan un modelo económico establecido y por tanto no son tenidas en cuenta por las instancias gubernamentales y organismos multilaterales que hacen parte del discurso hegemónico eurocéntrico y occidental.

Ahora bien, también hay una relativa y reciente fundamentación iusfilosófica (Llanos, 2013) que intenta dar respuesta a los interrogantes que la doctrina del derecho internacional ha planteado como 
desafíos de cara al desarrollo, pero desafortunadamente ésta no lo concibe desde la ética y menos se pregunta por el núcleo esencial. Por tanto, el derecho al desarrollo continua considerándose por la mayoría de los estudiosos, como una síntesis de los derechos humanos, que en el mejor de los casos es comprendido como un derecho autónomo e interdependiente con los demás derechos humanos; y en el peor de los casos, algunos refieren que no debe ser considerado como derecho humano, pues hace que pierdan vigencia los derechos civiles, políticos, económicos, sociales y culturales. También hay disenso entre si es un derecho de tercera o cuarta generación, en razón a su aparición reciente dentro del catálogo de los derechos humanos. A pesar de todo ello, consideramos que el derecho al desarrollo sigue sin ser abordado en lo fundamental, es decir, carece de una fundamentación ética y núcleo esencial. Por tanto, consideramos oportuno partir de la base de que el derecho al desarrollo se encuentra en todos los derechos y todos los derechos están en este derecho.

Teniendo en cuenta lo anterior, y en la relativa carencia de fundamentación teórica del derecho al desarrollo, nos hemos preguntado: ¿De qué manera se fundamenta el derecho al desarrollo, teniendo en cuenta el desarrollo de todos los seres que integran e integrarán la Comunidad de la Vida?

Por tanto, lo que hemos planteado como objetivo central ha sido proponer una fundamentación ética y el núcleo esencial del derecho al desarrollo desde la complejidad y la biociudadanía.

Lo antes dicho, responde a que queremos presentar una manera diferente de sentir y pensar el derecho al desarrollo, movidos por una comprensión distinta acerca del desarrollo, partiendo desde la óptica del ser vivo que integra la Comunidad de la Vida.

El enfoque y método de esta investigación es cualitativo y hermenéutico, este último se fundamenta en la observación resultante de la interpretación, la cual se ha orientado hacia la identificación y análisis de significados y conceptos acerca del desarrollo y sus alternativas. Lo mismo con el derecho del desarrollo y el derecho al desarrollo, lo escrito alrededor de la fundamentación iusfilosófica del derecho al desarrollo, el antropocentrismo y el biocentrismo, al igual que todo aquello relacionado con la complejidad, el pensamiento complejo y la ética de 
la complejidad, de tal manera que se comprenden de una mejor manera manteniendo presente el contexto en el que se relacionan. La metodología seguida es la de investigación documental, que en un primer momento y con una revisión exhaustiva de fuentes bibliográficas primarias, nos permitió adentrarnos al estudio de los conceptos antes mencionados. Dicha revisión documental se realizó en artículos científicos consultando las bases de datos abiertas y cerradas afines a las temáticas de la investigación, con el fin de identificar la producción académica en revistas indizadas sobre dichos conceptos. Los hallazgos de los artículos y publicaciones se procesaron de tal manera que permitieron generar delimitaciones conceptuales en cada una de las categorías, derivar puntos de encuentro y desencuentro, y análisis relacionales con los que se ha propuesto el concepto de biociudadanía y el núcleo esencial del derecho al desarrollo de la Comunidad de la Vida.

Ahora bien, por cuestiones metodológicas se ha organizado de manera deductiva la presentación de este libro, y lo describiremos como una flor de loto que se compone de tres partes y cada capítulo será un pétalo. En la primera parte podremos apreciar dos capítulos, en el primero; analizaremos las diferentes nociones que han demarcado el camino del desarrollo desde sus inicios hasta nuestros días, incluyendo las alternativas a este concepto; revisaremos las diferentes posiciones que se han suscitado desde mediados del siglo pasado para tener una panorámica completa de esta noción, comenzando con una visión tradicional, que luego empieza a despertar la moda verde de la sostenibilidad, el desarrollo humano y una creciente ola de iniciativas opuestas al modelo de desarrollo imperante, bajo el título del posdesarrollo, incluso incursiona una nueva visión denominada transdesarrollo, entre otras-. Algunas de estas propuestas corresponden a cosmovisiones de pueblos y culturas que deberían ser escuchadas, pues contribuyen al delineamiento de aquello que comprendemos por desarrollo, y del cual haremos mención extensa.

El segundo capítulo trata del surgimiento del derecho al desarrollo, que se encuentra dentro de los nuevos derechos o derechos emergentes, junto con sus críticas. Revisaremos lo que se comprende por derecho del desarrollo y derecho al desarrollo, para avanzar con el análisis respecto al reconocimiento del derecho al desarrollo como derecho humano en 
la doctrina y por la comunidad internacional, presentando las críticas y observaciones de su reconocimiento en la Declaración sobre el Derecho al Desarrollo (1986), y de la que nos serviremos para estudiar la titularidad, los deberes y la configuración conceptual de este derecho.

En la segunda parte, encontraremos los capítulos tercero y cuarto, teniendo en cuenta que el derecho al desarrollo adolece de una adecuada fundamentación ética que permita dilucidar su conceptualización, de cara a la materialización del desarrollo y del derecho que se desprende de éste, en el que apreciamos cierto silencio prolongado, observando una descolorida y tenue atención por hallar alternativas diferentes que sirvan de base y cimentación a un derecho emergente, como lo es el derecho al desarrollo, en el capítulo tercero podremos observar y analizar qué se ha planteado actualmente acerca de la fundamentación iusfilosófica del derecho al desarrollo. Para ello, el profesor Fernando Llano nos presenta la articulación y armonización de ideas encaminadas a contribuir a la fundamentación del derecho al desarrollo a partir de autores como Immanuel Kant, John Rawls, Amartya Sen, Martha Nussbaum, entre otros ${ }^{2}$ con los que ha construido su argumento, resaltando la idea de dignidad humana, el libre desarrollo de la personalidad, el desarrollo como expansión de las libertades y la creación de capacidades, todas ellas con sus aspectos relevantes y críticas de suma importancia, y las contribuciones científicas que nos ayudan a transcender y continuar el camino, para enfocar la mirada hacia el bioantropocentrismo como base ética del derecho al desarrollo.

El capítulo cuarto, denominado "La espiral de Arquímedes. Antropocentrismo, biocentrismo, responsabilidad y solidaridad" está compuesto a su vez de dos secciones, en la primera veremos la importancia de la fundamentación ética y la evolución que desde la ética medioambiental o ética ecológica —empleando cualquiera de los dos términos indistintamente, toda vez que observamos que en cada una de estas existe un mayor o menor grado de comprensión respecto

2 De otra parte, el profesor Llano ha continuado su explicación hacía la doctrina universalista cosmopolita, pero como esta empresa desborda el objeto de nuestro trabajo debemos decir que su análisis no será abordado. 
a la relación moral que nosotros debemos mantener con la naturaleza-, fue necesario que realizáramos la revisión de diferentes planteamientos que se han dado, acerca de las éticas derivadas de la relación entre la naturaleza y el ser humano en aras de responder a su fundamentación, pues consideramos que esta situación ha sido vista desde una sola óptica. Es decir, desde el antropocentrismo. Por tanto, de una manera diferente mostraremos la relación ética entre la Madre Tierra y el ser humano, y las distintas comprensiones que se han suscitado en favor y en contra de la ampliación de la comunidad moral a seres no humanos, presentándolo a partir de una espiral que inicia desde el antropocentrismo fuerte o extremo, pasando luego al antropocentrismo débil, siguiendo al biocentrismo débil o moderado, en el que hacemos un viraje al biocentrismo fuerte o extremo con sus diferentes matices, e incluyendo las miradas acerca de la consideración moral. Mientras que en la segunda sección podremos analizar lo relacionado con la responsabilidad y la solidaridad, viendo los puntos de vista de diversos autores que permiten hacer una exposición amplia y relacional entre naturaleza y ser humano, y la importancia de estos dos valores en la construcción tanto de la visión del desarrollo como del derecho al desarrollo.

La tercera parte, denominada "Complejidad, pensamiento y ética compleja: fundamento ético del derecho al desarrollo", está integrada por el capítulo quinto y sexto. En cuanto al capítulo quinto nos ubicaremos en la complejidad y pensamiento complejo, sus críticas, la dialéctica del medio de Françoise Ost, la unidad dialéctica y el principio femenino de Vandana Shiva, para avanzar en el saber pensar, que además se nutren de la ética del pensamiento complejo de Edgar Morín, aspectos que consideramos pueden ser fundamento ético del derecho al desarrollo de la Comunidad de la Vida desde la complejidad.

El capítulo sexto titulado "Biociudadanía y núcleo esencial del derecho al desarrollo", está compuesto por dos secciones. En la primera presentaremos el concepto de biociudadanía con el objetivo de personificar la ética del pensamiento complejo que contribuye a consolidar aún más la fundamentación ética del derecho al desarrollo y a su realización. Aprovechando las aportaciones de los autores antes mencionados, algunos aspectos de la física cuántica y otras contribuciones de 
la ciencia contemporánea como las neuronas espejo, y especialmente la visión psicológica de Jiddu Krishnamurti, se propicia un diálogo armónico de cara a la construcción del concepto propuesto, y con ello potenciar la realización del desarrollo y por tanto el derecho al desarrollo de la Comunidad de la Vida. En la segunda sección planteamos la propuesta de núcleo esencial del derecho al desarrollo, con el fin de contribuir a la configuración conceptual de este derecho desde los principios medulares del pensamiento complejo. En este sentido, hacemos referencia a diferentes posiciones que han abordado lo concerniente al contenido esencial de un derecho. Asimismo, de la sentencia 11/1981 del 8 de abril del Tribunal Constitucional de España y la sentencia C-756 de 2008 de la Corte Constitucional de Colombia, tomamos los elementos más destacados, que han realizado juiciosamente respecto al núcleo esencial. En especial nos serviremos de lo mencionado en la decisión de esta última Corte, la cual ha expresado con claridad la definición y rasgos característicos que contribuyen a identificar el núcleo esencial de un derecho. Lo cual deviene en definirlo como derecho complejo e implica una nueva manera de valorarlo de cara al catálogo de derechos, y la relación naturaleza-ser humano que explica la titularidad y la manera en la que el ser humano puede hacer posible que el desarrollo se realice en la Comunidad de la Vida, y que desde luego nos conduce a asumir deberes y el reconocimiento del estatus jurídico como derecho al desarrollo de la Comunidad de la Vida, pues con ello pretendemos dar un salto cuántico para salir de la encrucijada discursiva y aisladora a la que se le ha sometido durante varios años con precarios frutos prácticos.

Derivado de lo anterior, presentamos dos criterios para tal fin; por una parte denominados de identificación y caracterización, en el que hemos analizado la titularidad, deberes y estatus jurídico a partir de los tres principios del pensamiento complejo de Edgar Morin; dialógico, recursivo organizacional y hologramático, con los cuales explicamos el núcleo esencial del derecho al desarrollo y; por otro lado, de realización y manifestación, a partir de cuatro elementos esenciales; vida, libertad, creatividad y amor, teniendo en cuenta también la creación de capacidades de las que nos habla Martha Nussbaum. 
Con todo ello, expondremos los principios que permiten identificar a este derecho y lo definen como complejo, y mencionaremos los elementos que permiten la realización de este derecho no solamente desde la perspectiva de la biociudadanía, sino también de todos aquellos seres que hacen parte de la Comunidad de la Vida, para que el derecho al desarrollo se realice en el desarrollo, que desde luego contribuye a esgrimir que a todos aquellos seres que hacen parte de la Comunidad de la Vida también les asiste el derecho al desarrollo. Al cierre, presentamos las consideraciones finales de la investigación y un epílogo artístico denominado biociudadanía.

Corolario de lo anterior afirmamos que el alcance de la fundamentación ética y la formulación del núcleo esencial del derecho al desarrollo le permite a este derecho, sea realizado cuando el sujeto activo/ observador consciente, crea la realidad del desarrollo y la manifiesta desde la biociudadanía, como en efecto lo expondremos, y en razón a ello, reivindicamos el fundamento ético planteado y el núcleo esencial del derecho al desarrollo. Con lo cual pretendemos mostrar que el condicionamiento y mecanicismo en cada ser humano que se ha convertido en una inconciencia colectiva, pueda cambiar para hacerse consciente de manera progresiva, y lograr otra comprensión y manera de relacionarse con la Comunidad de la Vida y el Estado.

Como podremos ver, la propuesta ha surgido como alternativa a la relativa ausencia de una fundamentación ética y del núcleo esencial del derecho al desarrollo, en aras de que a su vez tenga una mirada compleja en relación con los seres que habitamos y habitarán en la Comunidad de la Vida y contribuya a que el desarrollo se realice.

En razón a ello, es necesario resaltar que hemos intentado integrar armónicamente conceptos y estudios científicos de diferentes áreas del conocimiento, haciendo de ellos una exposición que facilite su comprensión con la visión del paradigma del pensamiento complejo de Edgar Morin que, entre otras cosas, ha sido de suma importancia para el desarrollo de esta propuesta.

En este sentido la investigación si bien resalta el valor de cada ser vivo, pretende armonizar la relación que existe entre naturaleza y ser humano en la Comunidad de la Vida, en virtud de ello, este trabajo se orienta hacía el rescate de la libertad fundamental e interior de cada 
ser humano, para explicar a la persona como ser social partiendo de la comprensión de ser un todo en cada parte, es decir, que la persona hace parte de la Comunidad de la Vida, lo cual involucra indiscutiblemente una reflexión compleja de la vida que se sirve de la biomímesis, de cara a la construcción del sistema de relaciones en la citada Comunidad, y su correspondencia con el derecho al desarrollo, en razón al entorno natural y cultural del cual hace parte el ser humano.

Finalmente, este libro contribuye al desenvolvimiento de la discusión del desarrollo y a la fundamentación del derecho al desarrollo desde la complejidad. Además, abre caminos para repensar el papel de la comunicación en el otro desarrollo y el otro desarrollo en la comunicación. 